\title{
Research on Design and Implementation of Sports Equipment Management System
}

\author{
Mingxia Yang ${ }^{1, a}$, Liang Jiang ${ }^{1}$ \\ ${ }^{1}$ Qinhuangdao Institute of Technology, Qinhuangdao, hebei, China \\ aqhdymx@126.com
}

Keywords: Management System, Sports Equipment, Design and Implementation

\begin{abstract}
Currently, there are many problems in the management of sports equipment, therefore, develop a set of sports equipment aspects become inevitable, in which labor control system is took place by a system platform management processes. This article is mainly to meet the needs of information technology, the use of Microsoft's .NET technology based on B/S to realize the mode of sports equipment management system. Because systems analysis, design and code unified representation method based on object-oriented method can achieve a seamless link, so this object-oriented approach to model the system and uses UML modeling language to describe. The system is based on object-oriented model, using Visual Studio.NET 2008 development platform and SQL Server2005 database management system to realize the system and ultimately the formation of a Web application based on B/S mode. This paper will help to improve the efficiency of the management of college sports equipment and sports science universities can be given greater room for development, but also the University information system played a positive role in promoting the development of various universities.
\end{abstract}

\section{Introduction}

With the continuous improvement of science and technology, computer science continues to develop and its powerful features have been deeply understand that it has entered all areas of human society and play an increasingly important role. Universities sports equipment rental system is part of a university of its content management indispensable for sports equipment managers and those who borrow sports equipment is vital, therefore, universities and sports equipment rental system should be able to users and managers to provide adequate information and inquiry means fast, but has been the use of the traditional manual management of inventory, this management approach, there are many shortcomings, such as low efficiency, confidentiality and poor, but over time, will produce a large number of files and data, which for the search, update and maintain all brought many difficulties. With this new model, for the University to create a new educational environment that manage to break through the constraints of time, to expand the management scale, improve work efficiency and management level, so that sports equipment managers, teachers may at any time, any place through the network for management and information dissemination [1].

Universities sports equipment rental system focused on providing a simple and practical network management platform and accurate and reliable information for sports equipment managers, to provide a convenient and friendly and efficient service for sports equipment borrower. Universities Sports equipment rental system is mainly to meet borrow sports equipment and sports equipment management needs, through the application of sports equipment rental sports equipment management system to improve management efficiency, conservation-related management costs, standardize sports equipment management tools, to form a package in line with the research and design theory of modern sports equipment management, to meet the needs of modern sports equipment development. The theoretical value of the performance in the improvement and development of traditional sports equipment management system design theory and method.

The value of performance in the summary and the formation of theories and methods based on the network environment of sports equipment management system designed to optimize the management of sports equipment; explore and summarize the information age how to reform the 
traditional ideas and models, allowing users to learn to use network resources methods and management experience; develop universities sports equipment rental software has some practical value.

\section{The Architecture Design of Sports Equipment Management System}

Package Model of Sports Equipment Management System. Package diagram is used to group elements, he is a high-level diagram allows designers to design class diagram class grouping, and the ability to group related classes linked. Sports equipment management system in addition to the business logic classes, you also need some auxiliary classes to complete the system functions, these auxiliary classes include user interface classes and database support classes. Business logic classes included in the package business logic, user interface classes are included in the presentation layer package, database access classes included in the data manipulation package. Fig. 1 depicts the package diagram sports equipment management system [2].

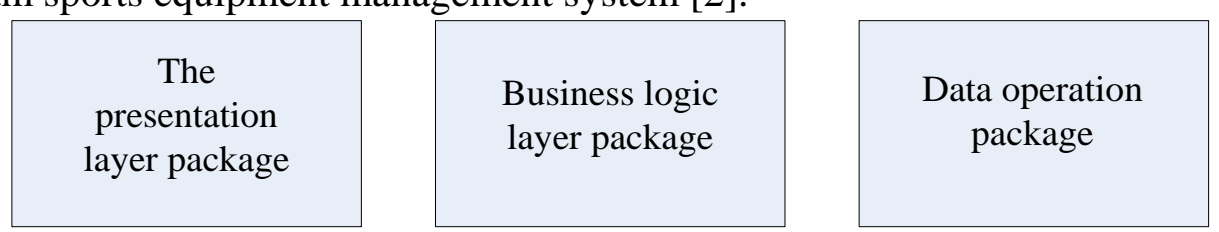

Fig. 1 The package graph of sports management system

Package diagram can also be nested to describe the different levels of the package, a package can contain several packages, which contain relationship can be used to describe the decomposition of system functions. Bag represents nested systems and subsystems, including the representation of a system that includes a number of sub-systems. In sports equipment management system, based on the functional requirements of the system and combined with the demand for sports equipment management system made by section analysis, to map out the business logic package diagram sports equipment management systems, business logic package contains user management packs and equipment management package, and equipment management Pack and management Pack from the storage, maintenance management package, waste management packs and circulation management packages.

Level Design of Sports Equipment Management System. Layering is the way the system effective organization. When the system uses a layered architecture, is the next layer on layer assembly component is responsible for providing services, the upper component can be used to define the underlying components of the service, but nothing about the underlying components of the upper component, typically between layers are mutually transparent, each layer has its own responsibilities. The more complex the system is into the layer systems is often more important. Over the above analysis, the system uses the three-tier architecture is the most appropriate. These three are: the presentation layer, business logic, and data layers [3].

Presentation layer: for the interaction between the user and information systems, is responsible for displaying information to the user and to obtain information or to request a variety of actions to explain the business logic layer or data storage layer from the user. This layer contains a presentation package.

Business Logic Layer: This layer contains all information systems and related work areas, including the input data or existing data calculation, input data from the presentation layer for authentication, and according to the command received from the presentation layer to determine which data should call layer logic.

Data Layer: This layer encapsulates the common functions used to perform database operations. Information extracted from the database, the filtered data is transferred to the business logic layer, the layer that contains a database access package. Fig. 2 shows the system level. 


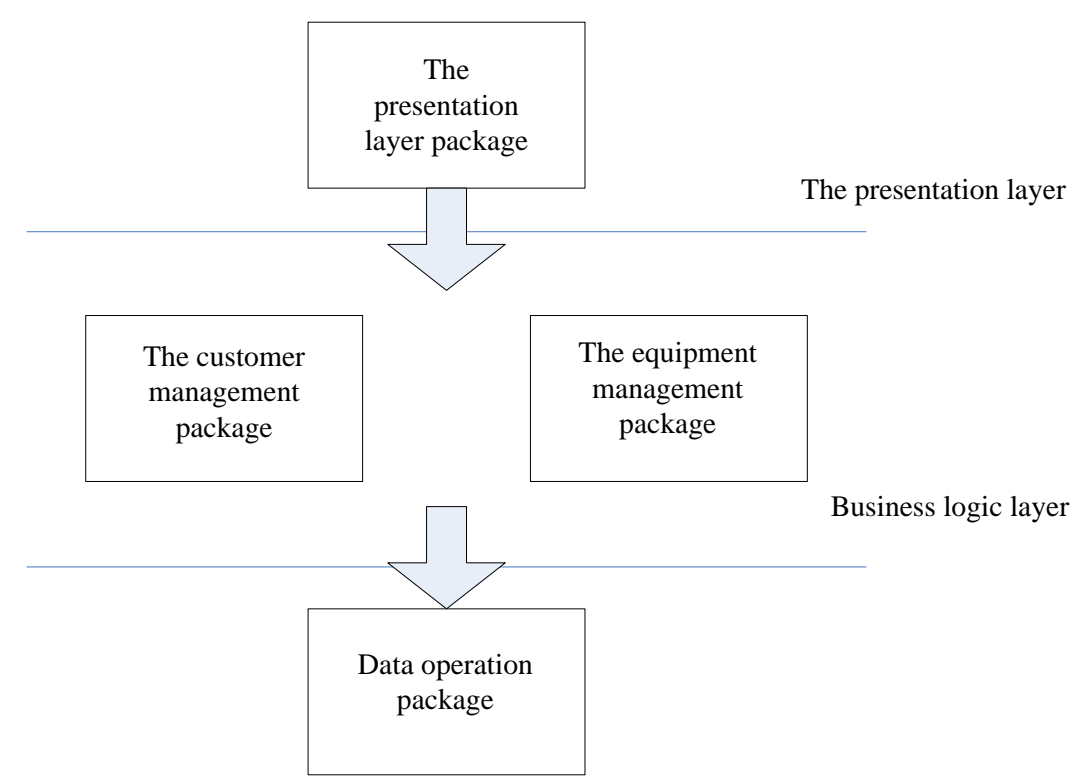

Fig. 2 The hierarchy graph of sports management system

\section{Module Design of Sports Management System}

According to the overall goal of sports equipment management situation analysis and sports equipment management system, the system function module roughly divided into foreground and background management module function modules. Reception function module includes the following sub-modules: View Account module, equipment module and modify the master of information modules. Management module also includes the following sub-modules: user management module, management module also borrow equipment and equipment management module [4].

\section{The Software Design of Sports Management System}

Software category is discussed in class design stage, also known as design classes. Requirements analysis phase is a discovery process, resulting in the analysis phase classes are usually abstract reality of things or concepts, we rarely care about the details of the properties and methods. In the design phase, we need to convert these conceptual class software world class, and in order to complete the functional use cases described, you must also add some field of computer software objects and classes.

Business logic layer including the front and back office management function module two, but they are also made up of several sub-modules, such as backstage management module is divided into user information management module, equipment management module. Control class represents the coordination, scheduling, and control of transaction processing other objects, are often used for packaging and related to a specific example of the control. Generally control the class into the business logic layer. In this system the major classes of software design, including: general user class, System Administrator class.

\section{The Implementation of Sports Equipment Management System}

The Implementation of System General Application. Database management system is inseparable from the participation in, and the connection and query the database to any management system is the most versatile features, the importance of database connectivity based on the system we package the basic class SqlHelper database operations, it provides a common set of database to perform a function, by its use, we can simplify the operation of the database connection operation, but also make the system more standardized code and simple and beautiful. Web page background program by calling the database command to access the database using ADO.NET 
(ActiveXDataObject.NET) technology, connect to a data source DB_SportsEquipment, perform the specified command to access the data source, such as select scripts rows or columns of data returned to the client end results, finished modifying the data, update, verify, delete and other operations, to achieve dynamic linking between pages. As with the call by the specific design of the database, the database table to achieve dynamic changes and updates, maintenance, and changes in the data used to store information page, making the data resource website to better serve users.

The Implementation of Reception Function Module. Reception function module mainly login module by the user, query account information module, modify account information modules and master of sports equipment module composed of four sub-modules. This introduces the user login module, master of the equipment module and information to modify the module [5].

The Implementation of Management Module. Management module is to realize the entire sports equipment management system design and focus on implementation. It includes a user information management module, equipment and materials information management module by module also three sub-modules, which also borrowed equipment module is focused on implementation. To ensure the safety of the system, the background of every operation there is a need user with appropriate privileges, even if the user is an administrator has landed. If the administrator user does not have the appropriate permissions, it still can not perform this operation. For example when carrying equipment circulation management, users need to have administrator privileges reception management.

\section{Conclusions}

This paper realized the function that sports equipment management system can assist managers in storage, circulation, abandoned library work; It check my account information and online through online master of ways to bring the convenience of ordinary users. Such a system can meet the different needs of different roles. Those equipments have changed the situation that traditional management modes are often low efficiency and poor accuracy, break through time and space limitations of traditional equipment management, expand manageable size and also actively promote the university's information technology construction. Of course, this system still exist deficiencies in the actual use of the process will inevitably find some issues need to be further tested and refined in practical work. I believe that through the amendments and improvements, sports equipment management system will provide better help for sports equipment management.

\section{References}

[1] Bruno Siciliano, Luigi Villani:Scientometrics, Vol. 22 (2006), No.3, p. 61- 62.

[2] Long Xi: Transactions on Robotics and Automation Vol. 4(2009), p. 42-58.

[3] S.C.Solanki, W.E.Dixon, C.D.Crane, S.Gupta: Journal of Beijing University of Technology, Vol. 1 (2011) p. 4-7.

[4] Zhang Songming: Journal of Beijing University,Vol. 2 (2006), p. 15- 24.

[5] Wang Xiangyong: Journal of Huazhong University of Sicence and Technology, Vol. 18 (2011) No.19, p.1-10. 